\title{
Data-Mining Application to Operating Room Service Performance in the Medical Center
}

\author{
Operating Room Service Performance
}

\author{
Hsueh-Ling $\mathrm{Ku}$ \\ Cathay Medical Center \\ National Central University \\ Ching Kuo Institute of Management and Health \\ Taipei, Taiwan ROC \\ 201385@cgh.org.tw
}

\author{
Jen-Der Leu \\ National Central University \\ Taoyuan, Taiwan ROC \\ leujunder@mgt.ncu.edu.tw
}

\begin{abstract}
Operation rooms are the most critical medical treatment unit in a surgical department, to which high costs of labor and resources are devoted. Managing the resources of operation rooms is just like those in the usual enterprises, both of which need to make good use of all the software and hardware; in addition, they should have the ability of providing competitive-advantaged services. Our research used total 21, 734 records of surgical patients' length of staying time in an operation room in 2009 in a certain medical center. With the method of Data-Mining-based statistical analysis, we found that the important factors, which affect the forecast of service performance indicator, include the expenses of operations and anesthesia, and the time of operations, etc. In the meantime, we also found that whether or not the surgical patients received general anaesthesia is the most important affecting factor, and the complexity of the operation is the critical factor of the variation in operation time. The resault of this study can be regarded as the points of scheduling tactic, which need to be improved at the operation in the hospital to promote the service efficiency of operation scheduling for surgical patients at the hospital.
\end{abstract} Mining

Keywords-Operating Room; Service Performance; Data-

\section{INTRODUCTION}

There are a large number of precision instruments in the operation room, $9 \sim 10 \%$ of the resource budgets are devoted there, and in the meantime, it is also a high-professionalintensive center [1].To manage the resources of operation rooms is just like those in the usual enterprises, both of which should have the ability of providing competitiveadvantaged services. Thus, the following problems should be considered, including how to arrange patients into the schedule of operation rooms in one day, how to make use of order and schedule to reach the particular requests, and how to evaluate the quality of schedule [2].There are many differences between the management of hospital and manufacturing industry. In the aspect of operation scheduling, because there are usually emergent patients in the hospitals, and the individual differences among each patients are quite huge, usually surgeons are not able to know how many operation procedures they should proceed until the operation be performed. Therefore, the practical and prearranged operation performance will be very different $[3,4]$. The progress of patients' disease is highly uncertain, so to develop correct and more representative simulate mode is still a developing research topic. For this reason, in the aspect of procedure-reforging, there is still a quite big development space in how to maximize the operation yield of every unit, how to minimize the using time of operation rooms, and how to reduce the berden of medical resources [5].

Litvak and Bisognano have found that although the hospital is full of people, the usage rate of beds is still low. They believed that the total yield of hospitals can be improved through appropriate managing, and the quality of medical services will thus rise in the meantime. The result of the study indicated that the usage rate of beds can be raised up to $80 \%$ $90 \%$ in a condition of not increasing beds and personnel through adjusting and redesigning patientcaring procedures [6]. Dexter et al. also found that to cut down the turnover time can lower the labor cost remarkablly after looking into medical records in four teaching hospital; it is not because of the reduced overtime of personnel but because of the reduced turnover time in the operation room [7]. Although operation scheduling is similar to production planning, operations are hardly performed according to the plan in the end because there are a lot of emergent cases of operation; in addtion, conditions and operation procedures are usually uncertain until the operation started, and even if the same operation procedure should be performed, the operation time will differ from surgeons, patients or the operation process $[3,4]$. These problems mentioned above cause the management of operation scheduling to have the variability of requirement, the ability of reacting differently to the health system, the ability of evaluating the usage efficiency of facilities, the ability of analysing the cost benefit, the evaluation of patient-caring results, and the ability of evaluating the degree of patient-caring satisfation, etc.

The method of Data-Mining-based statistical analysis not only allows the abudant data to be handled by using the traditional algorithm, but also creats the methods of analysing new-pattern data. There are four main techniques 
of data-mining: prediction model, clustering analysis, abnormality detection, and association rule analysis. The aim of the prediction model is to set up a mode of learning, hoping the learning mode can minimize the error between the predicted values and the actual data. Clustering analysis is a method to find out the observation values with similar characteristic and those with different characteristic from other groups. Abnormality detection is to find out some observation values with the noticeable differences from a group of data, and these different observation values is exactly the so-called anomaly or outlier. And the association rule analysis is used to find out the high-related pattern among all the properties and charateristics from the data and to show them in an associated way.

Operation rooms play important roles in a hospital and daily operation scheduling is especially the impetus to the whole practices as engine. A high-quality operation scheduling system can provide patients with proper health caring immediately, raise the degree of patients' satisfation, the efficiency of whole medical teams and hospital operation in the meantime. Thus, it is a topic worth for operation managers to probe into by showing the historical data of opertaion process with statistical method, doing the cross match, analysis and management, finding out the crucial influential factor, and adjusting the order of operation scheduling Ease of Use.

\section{METHODS}

A. A brief introduction of the targeted hospital.

The targeted hospital in this research is evaluated as a teaching hospital and a medical center. There are totaling 772 sickbeds in the hospital, including 598 general sickbeds and 174 special sickbeds. The personnel of the hospital totals 1,758, including 349 doctors, 872 nursing staff and 537 health care professionals (the statistic was finished in December, 2009) The load of operation rooms in the targeted hospital is 21,734 people per year, and 70 people per day in average (30 people for outpatient clinic and about 40 people for admission and emergency.) There are 8 operation rooms in common use, including 4 rooms respectively mainly for the operation of ophthalmology, otolaryngology, plastic surgery and obstetrics-gynecology, 2 rooms for cardio-thoracic surgery and 2 rooms for any departments. Before improving the effects of operation rooms, the indicator and the influential factor of operation effects should be found in order to proceed the improvement by directing at the crucial factors to meet the maximum of benefit. Thus, the study expects to find out the "crucial influential factors" with the method of data analysis of datamining.

\section{B. Statistical analysis}

The study is analysed based on " Taiwan National Health Insurance Code". Using the data of hospital operation rooms from January 1st, 2009 to December 31st, 2009 , totaling to 21,734 data, in order to meet the closer methods of parameter input and estimation to the state of play, we contrasted our data with variables found in the documents first, and then switched and synthesized methodically the hospital data to the variables which can be supported by the documents, and began analysing and comparing by the method of statistical analysis based on data-mining in the end.

\section{Data processing and analysis}

\section{1) Data collection}

The records of operation rooms includes the basic information and conditon of patients (including whether or not the patients receive emergency operation, whether or not the patients are hospitalized,and patients' wards), name and the department of doctors, names of anesthesiologists and nursing staff, the length of scheduled time before the operation starts, the actual starting and ending time of the operation, and the kinds of operation performed.

\section{2) Data processing and analysis}

Sperating surgical data according to each department can not only figure out the average value, standard deviation, and the coefficient of variation of every operation time, but also crunch the number of people and the total operation time of every department. (Table 1 and Fig. 1,2)

TABLE I. Statictics on Operation Time of EACh Department

\begin{tabular}{|c|c|c|c|c|c|c|}
\hline Item & \begin{tabular}{|l|} 
Departme \\
nt
\end{tabular} & \begin{tabular}{|l} 
Averag \\
e \\
Value \\
\end{tabular} & $\begin{array}{l}\text { Standard } \\
\text { Deviation }\end{array}$ & Variation & Case-No & $\begin{array}{l}\text { Operatio } \\
\text { n Time }\end{array}$ \\
\hline A1 & Kidney & 85 & 0 & 0 & 1 & 85 \\
\hline B1 & General & 77.68 & 59.4 & 0.764 & 4806 & 373335 \\
\hline B2 & Cardic & $\begin{array}{c}147.3 \\
9\end{array}$ & 147.7 & 1.002 & 512 & 75465 \\
\hline B3 & Injury & 70 & 71.7 & 1.024 & 6 & 420 \\
\hline B4 & URO & 60.49 & 61.6 & 1.019 & 2115 & 127935 \\
\hline B5 & Colon & 27.95 & 44.9 & 1.605 & 2311 & 64599 \\
\hline B6 & Throcis & $\begin{array}{c}115.8 \\
4\end{array}$ & 86.6 & 0.748 & 290 & 33595 \\
\hline B7 & Ortho & 72.14 & 70.8 & 0.981 & 2841 & 204963 \\
\hline B8 & Neuro & $\begin{array}{c}161.9 \\
5\end{array}$ & 103.3 & 0.638 & 1069 & 173120 \\
\hline B9 & Plasty & 58.85 & 79.9 & 1.357 & 2649 & 155893 \\
\hline $\mathrm{C} 1$ & Obs-Gyn & $\begin{array}{c}123.3 \\
6 \\
\end{array}$ & 65.2 & 0.528 & 1131 & 139525 \\
\hline D1 & Oral & $\begin{array}{c}292.4 \\
5 \\
\end{array}$ & 259.3 & 0.886 & 98 & 28660 \\
\hline D2 & Dent & $\begin{array}{c}234.1 \\
7 \\
\end{array}$ & 79.6 & 0.34 & 18 & 4215 \\
\hline D3 & ENT & 81.11 & 84.8 & 1.045 & 1828 & 148260 \\
\hline D4 & Oph & 47.91 & 33.3 & 0.696 & 2059 & 98637 \\
\hline
\end{tabular}




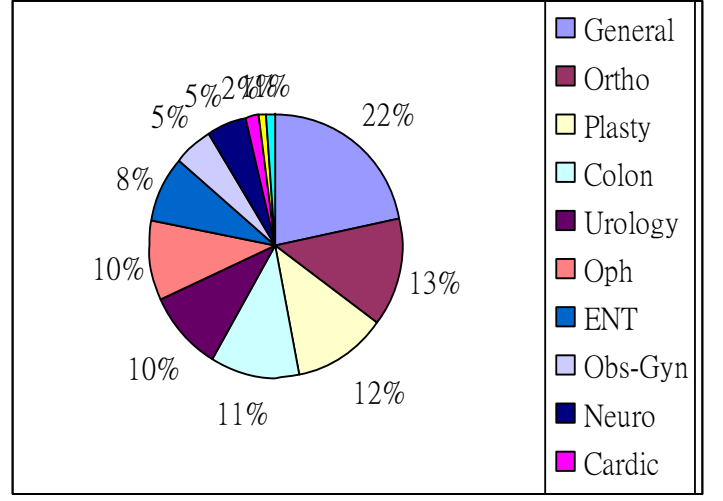

Figure 1. Distribution of the number of operations for each department

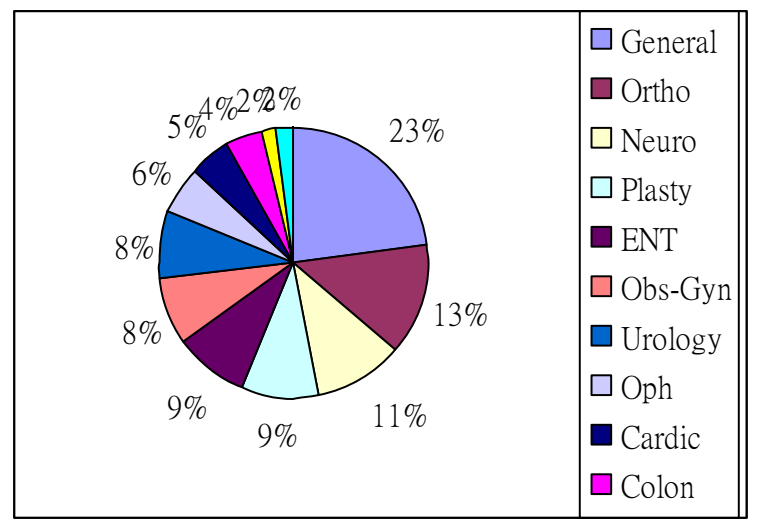

Figure 2. Distribution of total operation time.

\section{Methods of statistical analysis based on Data-Mining}

The study was completed by using IBM SPSS Modeler 14. software. Pieces of information were taken as the database for the study during the period from January $1^{\text {st }}$ to December $31^{\text {st }}$ in 2009.

\section{RESULT}

A. Data analysis on predicting the operation expenses

The most influential factor is whether or not the patients receive general anesthesia, and it is $42 \%$ of total; the following importance degree of factors in order includes $16 \%$ of whether or not the patients are hospitalized, $14 \%$ of whether or not the patients receive emergency treatment, $11 \%$ of the number of assistants, $7 \%$ of whether or not the patients receive emergent anesthesia, 5\% of the number of operation procedures, $3 \%$ of the seniority of anesthesiologist, $1 \%$ of the seniority of surgeons and $1 \%$ of whether it is the non-working day. (Fig. 3)

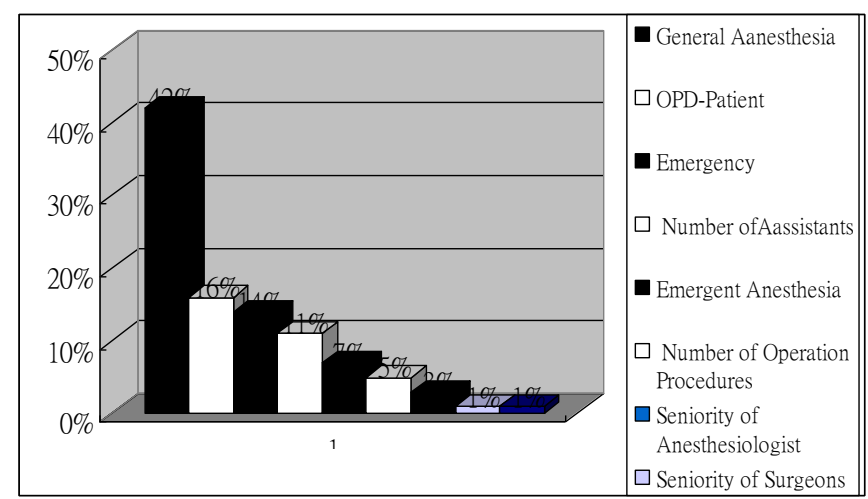

Figure 3. The importance degree of each factor of predicting the operation expenses.

$B$. Data analysis on predicting the anesthesia expenses

The most influential factor is whether or not the patients receive general anesthesia, and it is 59\% of total; the following importance degree of factors in order includes $26 \%$ of the seniority of anesthesiologist, $8 \%$ of whether or not the patients receive emergency treatment, $6 \%$ of whether or not the patients receive emergent anesthesia, and $1 \%$ of whether or not the patients are hospitalized. (Fig. 4)

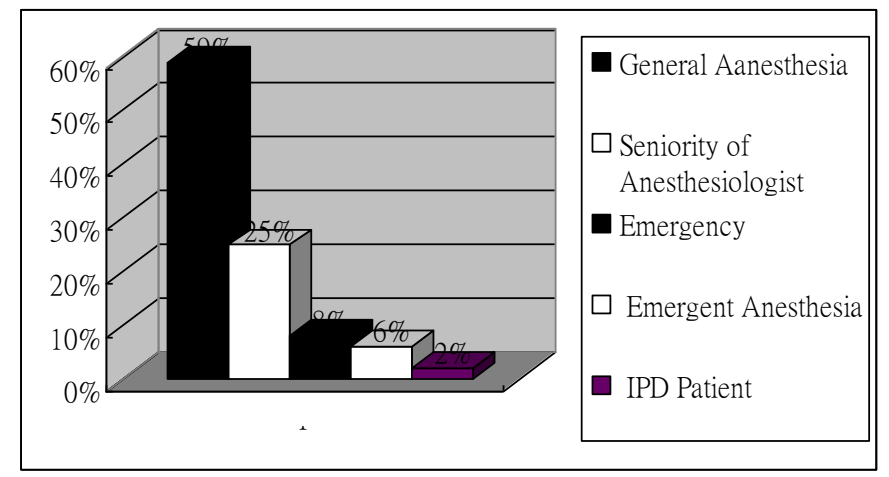

Figure 4. The importance degree of each factor of predicting the anesthesiaexpenses.

C. Data analysis on predicting operation time

The most influential factor is whether or not the patients receive general anesthesia, and it is $31 \%$ of total; the following importance degree of factors in order includes $26 \%$ of whether or not the patients are hospitalized, $22 \%$ of the number of assistants, $15 \%$ of whether or not the patients receive emergency treatment, $2 \%$ of the seniority of surgeons, $2 \%$ of whether or not the patients receive emergent anesthesia, and $2 \%$ of anesthesia. (Fig. 5.) 


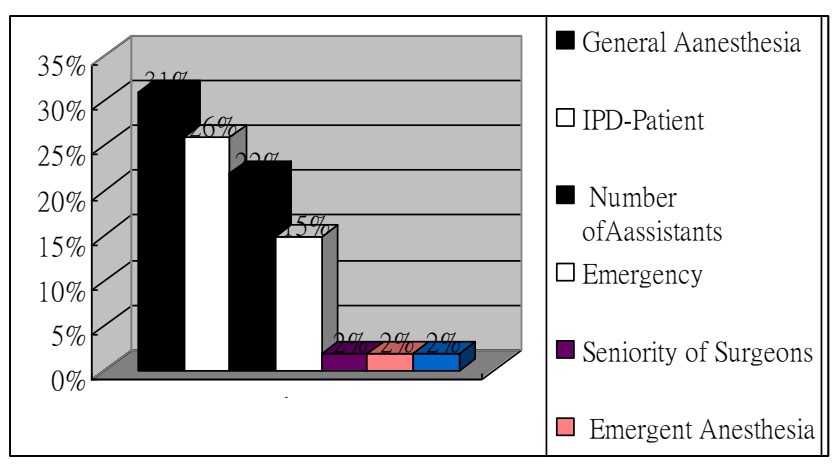

Figure 5. The importance degree of each factor of predicting the operation time.

$D$. Data analysis on predicting the variation of operation time

The most influential factor is the number of operation procedures, and it is $96 \%$ of total; the following importance degree of factors in order includes 3\% of whether or not the patients receive general anesthesia, and $1 \%$ of whether or not the patients are hospitalized. (Fig. 6)

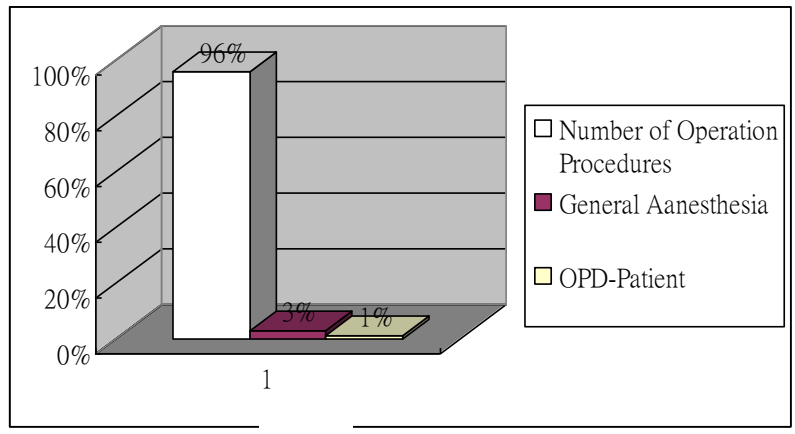

Figure 6. The importance degree of each factor of predicting the operation time variances

\section{DISSCUSSION}

Evaluating the effects of the whole hospital management, in the aspect of revenue, $40 \%$ of which comes from surgical services, among which, sales of operation performance in the operation room are the biggest revenue source [8].The operation of operation rooms has a great influence on surgical sales. Because of the high labor cost and the expensive facilities put into the operation room, the efficiency of operation room management should be paid much attention to. The number of operations in a spacelimited operation room is based on whether it is in an effective managing condition. Operation scheduling management of every surgical patients in each operation rooms is the most crucial thing for the management of operation rooms; whether the operation scheduling management is good will affect not only the safety of surgical patients, but also the effects and operating costs of operation rooms directly. A good scheduling system enables surgical patients to receive proper care; in the meantime, not only will the usage efficiency of operation rooms, but also the operating efficiency rise. Thus, scheduling orders of daily operations at every operation rooms are the very important observational indicators.

This study indicates that after the cancellation of the anesthesia item in Taiwan national health insurance code, the characteristic of Taiwan national health insurance code has become similar to the international disease procedure code so the follow-up study can be analysed by using this method. Through this study, we found out the important factors which will affect the predicted indicators of effects; the most influential factor of the expenses of the operation and anesthesia, and the lengh of operations is whether or not the patients receive general anesthesia, and the degree of operation complication is the most influential factor of the variation degree of operation time. Thus, we suggest members of the hospital can take these into the consideration of scheduling order or the optimization of medical quality and operation scheduling procedure.

Surgical patients having no choice can only make the decision of having their disease cured through the operation. Whether or not the operation proceeds smoothly is of a great concern of the quality of patients' and their family members' life. Once surgical patients enter the hospital, they all expect for the perfect quality of all the services provided by the hospital and personnel, therefore, managers of operation scheduling in the operation room should achieve all the proceeds that surgical patients have to face by all means to satisfy patients' need.

\section{CONCLUSION}

In addition to safety, to manage the operation schedule and the logistic of instruments is also important for the management of operation rooms. In order to reach the biggest benefit, by analyzing hospital's 21,734 data of operation room from January $1^{\text {st }}$ to December $31^{\text {st }}$ in 2009 , this study found that the most influential factor when predicting the variances of operation time is the operation procedures; on the other hand, whether the patients receive general anesthesia has the highest degree of importance when predicting the expenses of operation and anesthesia and the length of operations. The study develops a datamining-based method of statistical analysis by using the data resources of historical records of operation rooms in the hospital to be the suggestion when deciding the supportive scheduling system, expecting to monitor the operation rooms and the distribution, mean value, and coefficient of variation of the length of every operation, and then applying these to the decision of daily operation scheduling to promote the service efficiency and the proper usage of medical resources.

Operation rooms are the operating center of hospitals, which is in the top principle of "safety" and "efficiency". In the aspect of efficiency, we regard the high turnover rate of operation scheduling as the ultimate goal of management because the operating cost is high at the operation room, and either the dispatch of personnel or the turnover of instruments and patients are the influential factors of time and the cost; it is the key point to have an effective management for good hospital operating effects. By 
analyzing the data of the whole hospital, the following study is suggested to analyze and compare with the data of every department with the same model and provide the influential factors more precisely by directed against the application of operation scheduling in every department; in the meantime, if the hospital can provide the data of the turnover time between two operations, we will be able to not only have a deeper analysis for the turnover time, but also provide a more precise suggestion of the whole operation room management.

\section{ACKNOWLEDGMENT}

The completion of this study is attributed to the support of Superintendent Huang and Vice Superintendent Li, the assistance of the Chief of Medical Information Liu, RungMao and all operating room personnel. This project was supported by the Joint Research Fund (project number MR99011 CGH) of the Cathay General Hospital.

\section{REFERENCES}

[1] Viapiano, J. and Ward, D. S., 2000.Operating Room Utilization: The Need for Data, International anesthesiology clinics, 38(4), 127-140.
[2] O'Neill, L., \& Dexter, F. (2007). Tactical increases in operating room block time based on financial data and market growth estimates from data envelopment analysis. Anesth Analg, 104, 355-368.

[3] Eijkemans, M. J. C., van Houdenhoven, M., Nguyen, T., Boersma, E., Steyerberg, E. W., \& Kazemier, G. (2010).Predicting the Unpredictable A New Prediction Model For Operating Room Times Using Individual Characteristics and the Surgeon's Estimate. Anesthesiology, 112, 41-49.

[4] Strum, D. P., Sampson, A. R., May, J. H., \& Vargas, L. G. (2000). Surgeon and type of anesthesia predict variability in surgical procedure times. Anesthesiology, 92, 1454-1466.

[5] May, J. H., Spangler, W. E., Strum, D. P., \& Vargas, L. G. (2011) The Surgical Scheduling Problem: Current Research and Future Opportunities. Production and Operations Management, 20, 392-405.

[6] Litvak, E., \& Bisognano, M. (2011). More patients, less payment: increasing hospital efficiency in the aftermath of health reform. Health Aff (Millwood), 30, 76-80.

[7] Dexter, F., Abouleish, A. E., Epstein, R. H., Whitten, C.W., \& Lubarsky, D. A. (2003). Use of operating room information system data to predict the impact of reducing turnover times on staffing costs. Anesth Analg, 97, 1119-1126.

[8] Rutter, T, and Brown, A., 1994. Contemporary Operating Room Management, Advances in Anesthesia, 11, 173-214. 